\title{
Occurrence, Related Factors and Prognostic Value of Vascular Lake in Hepatocellular Carcinoma Patients Treated with Drug-Eluting Bead Transarterial Chemoembolization
}

\author{
Hao Li* \\ Manzhou Wang* \\ Pengfei Chen \\ Fangzheng $\mathrm{Li}$ \\ Donglin Kuang \\ Xinwei Han (D) \\ Jianzhuang Ren \\ Xuhua Duan
}

Department of Interventional Radiology, The First Affiliated Hospital, Zhengzhou University, Zhengzhou, People's Republic of China

*These authors contributed equally to this work

\begin{abstract}
Purpose: The present study aimed to assess the prevalence of vascular lake (VL), its associated factors and correlation with prognosis in hepatocellular carcinoma (HCC) patients treated with drug-eluting bead transarterial chemoembolization (DEB-TACE).

Patients and Methods: A total of 286 primary HCC patients (with 384 treated nodules) receiving DEB-TACE treatment were recruited, and their clinical characteristics were documented. The occurrence of VL was recorded, and treatment responses were assessed according to the modified response evaluation criteria in solid tumor (mRECIST).In terms of treatment response, the total response status (including CR, PR, SD and PD), objective response rate (ORR) and disease control rate (DCR) were elevated in VL patients compared to non-VL patients as well as in VL nodules compared to non-VL nodules. Liver function indexes and adverse events were assessed. Progression-free survival (PFS) and overall survival (OS) were evaluated with the last follow-up date of March 2020.
\end{abstract}

Results: The patient-based and nodule-based VL occurrence rates were $17.1 \%$ and $16.4 \%$, respectively. Larger tumor size, pseudocapsules and smaller bead size were independently associated with VL occurrence. PFS and OS were more prolonged in VL patients than in non-VL patients, and VL independently correlated with better PFS and OS. For liver function, the liver function indexes before and after DEB-TACE were of no difference between VL patients and non-VL patients. Additionally, the incidences of adverse events were similar between VL patients and non-VL patients.

Conclusion: VL occurs in $17.1 \%$ of HCC patients treated with DEB-TACE, and it is correlated with larger tumor size, pseudocapsule, smaller bead size, more favorable treatment response and better survival.

Keywords: hepatocellular carcinoma, drug-eluting bead transarterial chemoembolization, vascular lake, related factors, efficacy, safety

\section{Introduction}

Hepatocellular carcinoma (HCC), which accounts for most liver cancer cases, is one of the most lethal cancers and is predicted to result in 1 million related deaths worldwide in 2030. ${ }^{1,2}$ Patients with HCC are treated mostly according to the Barcelona clinical stage of liver cancer (BCLC) staging system. Potential curative therapies, such as resection and ablation, are recommended for patients at BCLC stage 0 or $\mathrm{A}$; however, patients at more advanced stages can only receive 
noncurative treatments, such as sorafenib, regorafenib, chemoembolization and supportive care. ${ }^{3,4}$ Unfortunately, patients at middle and advanced stages occupy the majority of HCC patients because the disease is mostly asymptomatic at early stages, which largely delays the duration before diagnosis. In addition, some early-stage HCC patients are not qualified for resection or other potentially curative therapies because of inadequate physical function or unique tumor features. ${ }^{5,6}$ Therefore, the management of these patients is crucial for improving the overall prognosis of HCC patients.

In recent years, chemoembolization has been increasingly applied in HCC patients at various stages, but the BCLC staging system only recommends it as a first-line therapy for intermediate-stage patients. ${ }^{7}$ Conventional transarterial chemoembolization (cTACE) is the leading type of chemoembolization until the existence of drugeluting bead TACE (DEB-TACE), which allows HCC patients to have more choices of treatment. More recently, studies have revealed that DEB-TACE not only exceeds cTACE in reducing systemic toxicity but also realizes more favorable efficiency in HCC patients. ${ }^{8,9}$ For instance, a previous study has reported that DEB-TACE achieves a better treatment response and survival profile than cTACE in HCC patients with a history of multiple cTACE treatments. ${ }^{8}$ Furthermore, other studies have stated that DEB-TACE displays acceptable efficacy and tolerance when treating advanced HCC patients, indicating a potential wider application of DEB-TACE in the clinical setting. ${ }^{10,11}$

Vascular lake (VL) is a phenomenon that occasionally occurs in the embolization of HCC, and it normally presents as blocking in the tumor-feeding artery and pooling of the contrast agent during embolization. ${ }^{12}$ The occurrence of $\mathrm{VL}$ is not uncommon in $\mathrm{HCC}$ patients during DEB-TACE treatment according to several reports, with an estimated prevalence of approximately 10\%-20\%; however, the causes that generate VL are still under investigation. ${ }^{12,13}$ Several researchers have suggested that the generation of VL may be correlated with the rupture of the targeted tumor caused by a sudden stasis in the tumorfeeding artery, resulting in a rapid increase in local pressure. ${ }^{12}$ However, reports about the precise mechanism of VL in DEB-TACE or other chemoembolization procedures in HCC patients are quite scarce. More importantly, the most concerning issue of VL occurrence is whether it has an impact on the efficacy and safety of DEB-TACE in HCC patients. However, this issue is rarely investigated as previous literature mostly focuses on case reports of $\mathrm{VL}$ during DEB-TACE in HCC patients, and studies about the effect of VL on the efficacy and safety of DEB-TACE treatment in $\mathrm{HCC}$ are scarce. In addition, previous studies all have small sample sizes, and no study has been conducted to explore the correlation between VL and longterm prognosis in HCC patients treated with DEB-TACE.

Hence, the occurrence of VL, its associated factors and its correlation with prognosis in HCC patients treated with DEB-TACE were investigated in this study.

\section{Methods}

\section{Patients}

In total, 286 newly diagnosed primary HCC patients who were treated with DEB-TACE as first-line treatment in our hospital between August 2016 and December 2019 were recruited for this study. The inclusion criteria were as follows: (1) diagnosed with primary $\mathrm{HCC}$; (2) age within 18 to 75 years; (3) at least one measurable lesion as defined by the diameter of the lesion on helical computed tomography (CT) scan $\geq 10 \mathrm{~mm}$ according to the modified response evaluation criteria in solid tumors (mRECIST); ${ }^{14}$ (4) well-to-moderately preserved liver function as defined by the Child-Pugh stage A or B; (5) normal renal function and normal coagulation function (or coagulation dysfunction corrected after treatment); (6) proportion of liver tumors less than $75 \%$ of total liver volume; (7) no previous therapy for HCC and suitable for DEB-TACE treatment; (8) life expectancy more than 12 weeks; (9) Eastern Cooperative Oncology Group (ECOG) performance score $0 \sim 1$; and (10) regular follow-up. The exclusion criteria were as follows: (1) Child-Pugh stage C; (2) severe coagulation dysfunction that could not be corrected after treatment; (3) serum bilirubin $>51 \mu \mathrm{mol} / \mathrm{L}$, alanine aminotransferase $($ ALT) or aspartate aminotransferase $($ AST) $>5$ upper limit of normal (ULN); (4) portal venous shunt; (5) left ventricular ejection fraction (LVEF) $<50 \%$; (6) serious comorbidities (eg, hypertension and coronary heart disease) or history of psychiatric disease or severe allergies; (7) HCC patients candidate for liver transplantation, resection, or local thermal ablation; (8) pregnant or lactating women.

\section{Ethical Approval}

This study was approved by the Ethics and Scientific Trial Committee of the First Affiliated Hospital of Zhengzhou University and was conducted in accordance with the 
principles expressed in the Declaration of Helsinki and adhered to the standards set by the International Conference on Harmonization and Good Clinical Practice. All patients signed informed consents. The study was registered in the Chinese Clinical Trial Registry with a registration number of ChiCTR-IOR -17012159 .

\section{Clinical Data Collection}

The clinical characteristics of the patients were documented, including age, sex, etiology, tumor number, portal vein invasion, Child-Pugh stage and Barcelona Clinic Liver Cancer (BCLC) stage. In addition, nodulebased features, including tumor size, tumor location and pseudocapsule, were also collected and recorded. Importantly, due to the confidentiality of our hospital's policy, the raw data of our study was not allowed to be public, therefore the data would only be accessible via contacting the corresponding author (for scientific research use only).

\section{DEB-TACE Procedures}

The DEB-TACE procedure was performed under local anesthesia. The right femoral artery was punctured by the Seldinger technique, and a $5 \mathrm{~F}$ arterial sheath was then placed. A 5F RH catheter or a 5F Yashiro catheter was selectively inserted into the superior mesenteric and celiac trunk for undergoing hepatic artery angiography, outlining the hepatic artery anatomy, identifying the feeding arteries of tumors and evaluating portal vein patency status. The feeding arteries of the tumor were catheterized with a microcatheter using the superselective catheterization method followed by perfusion chemotherapy. Afterwards, CalliSpheres ${ }^{\circledR}$ Beads (100-300 $\mu \mathrm{m}$ or 300-500 $\mu \mathrm{m}$, Jiangsu Hengrui Medicine Co., Ltd., Jiangsu Province, China) loaded with arsenic trioxide or adriamycin were injected into the feeding arteries of tumors for chemoembolization. The preparation of CalliSpheres ${ }^{\circledR}$ Beads and drug loading were performed according to the method described in previous studies. ${ }^{15,16}$ The embolization was stopped when the near stasis observed in the feeding arteries of tumors. If a tumor blush was still observed after injection of the loaded CalliSpheres ${ }^{\circledR}$ beads, additional polyvinyl alcohol (PVA) particles with diameters of 350-560 $\mu \mathrm{m}$ were added. The embolization endpoint was the disappearance of the tumor blush under angiography. Postoperative routine liver protection and symptomatic treatments were administered for patients without other special treatments.

\section{Management of $\mathrm{VL}$}

According to a previous study, the VL was defined as focal, well-circumscribed and persistent retention of contrast material in various forms on the venous phase of angiography and was distinguished from a tumor blush. ${ }^{12}$ Figure 1A displayed the HCC tumor of a 52year-old male patient, and a partial response (PR) after the DEB-TACE treatment (Figure 1D). In the current study, the VL phenomenon occurred in the early arterial phase of angiography after embolization with drugloaded CalliSpheres ${ }^{\circledR}$ Beads. The VL phenomenon changed its size progressively with microsphere embolization, disappeared slowly and was still observed after the venous phase (example shown in Figure 1B). For the tumor-feeding arteries with the VL phenomenon, drugloaded CalliSpheres ${ }^{\circledR}$ Beads were continuously used for embolization. If the drug-loaded CalliSpheres ${ }^{\circledR}$ beads were depleted, PVA particles were further added for embolization until the VL phenomenon disappeared (example shown in Figure 1C). Routine blood examination was performed at 1 day after the operation in patients with VL to confirm whether the PVA particles led to further rupture and hemorrhage of the tumor. According to the occurrence of the VL phenomenon during DEB-TACE, patients were divided into $\mathrm{VL}$ patients and non-VL patients, and the nodules were divided into VL nodules and non-VL nodules.

\section{Assessments and Follow-Up}

All patients underwent contrast-enhanced CT or Magnetic Resonance Imaging (MRI) at 4 6 weeks after first DEB-TACE, and the treatment response for each nodule and patient was evaluated according to the mRECIST criteria, ${ }^{14}$ where the response was classified as complete response (CR), PR, stable disease (SD), and progressive disease (PD). Objective response rate (ORR) was defined as $\mathrm{CR}+\mathrm{PR}$, and disease control rate (DCR) was defined as $\mathrm{CR}+\mathrm{PR}+\mathrm{SD}$. Liver function was determined before DEB-TACE, one day after DEB-TACE, 7 days after DEB-TACE and 1 month after DEB-TACE. Adverse events after DEB-TACE, such as nausea, vomiting, abdominal pain and ascites, were recorded in detail and graded according to the National Cancer Institute Common Terminology Criteria for Adverse Events (version 4.0). In addition, patients were followed 

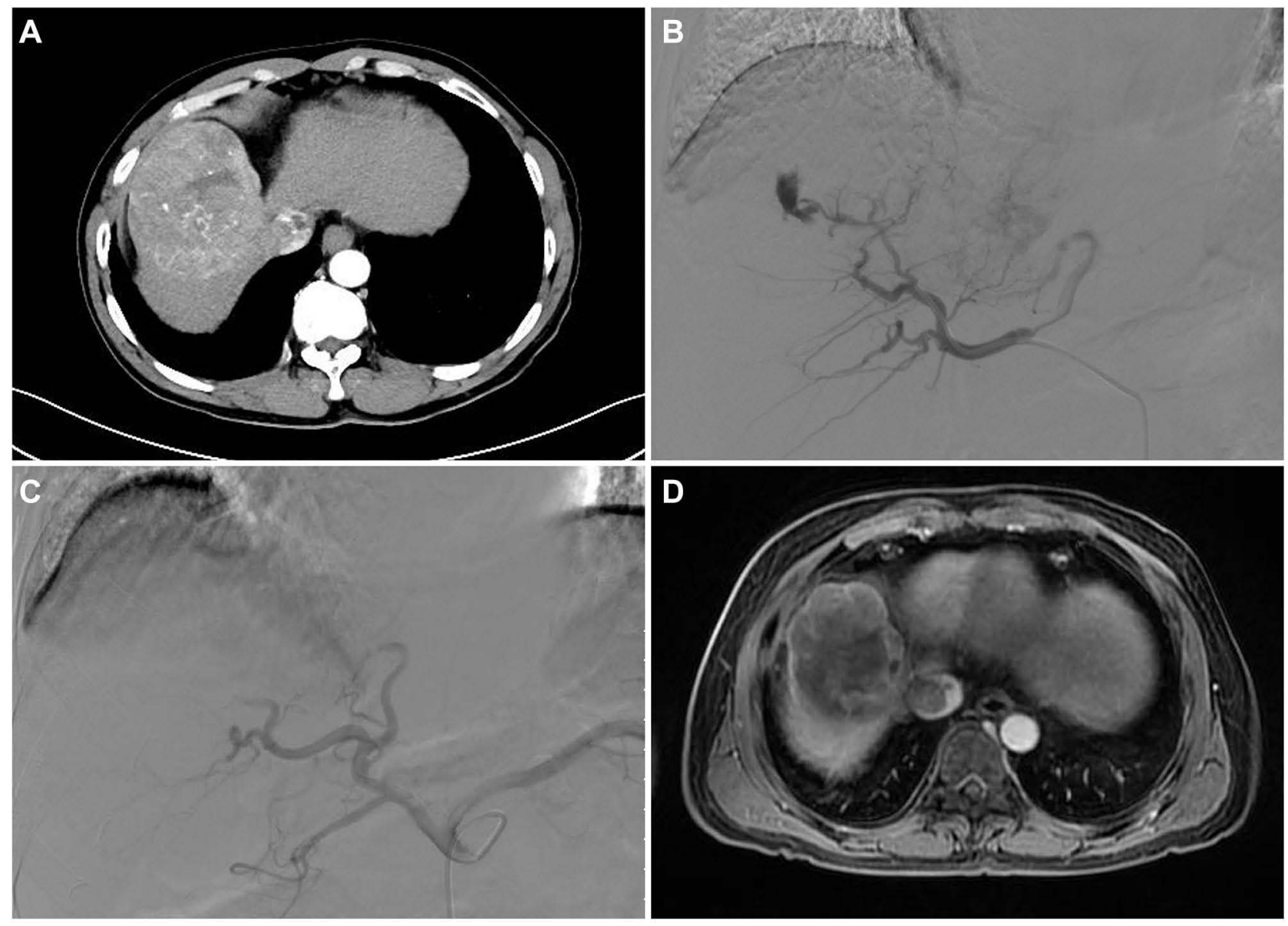

Figure I Image of VL and its management in a 52-year-old male patient. Arterial phase in the CT was significantly enhanced, and the middle hepatic vein and inferior vena cava were invaded by tumor (A). In the first DEB-TACE, CalliSpheres ${ }^{\circledR}$ Beads with a diameter of $100-300 \mu \mathrm{m}$ loaded with $15 \mathrm{mg}$ of arsenic trioxide were used to embolize the right hepatic artery, and the VL phenomenon occurred in the re-examined angiography (B). PVA particles with a diameter of $350-560 \mu \mathrm{m}$ were subsequently added for embolization until the VL phenomenon disappeared in the re-examined angiography (C). MRI at 4 weeks after first DEB-TACE revealed partial contrast enhancement in the peripheral of intrahepatic lesion, suggesting a partial response according to the mRECIST criteria (D).

Abbreviations: VL, vascular lake; CT, computed tomography; DEB-TACE, drug-eluting bead transarterial chemoembolization; PVA, polyvinyl alcohol; MRI, magnetic resonance imaging; $\mathrm{mRECIST}$, modified response evaluation criteria in solid tumors.

up every 4-6 weeks by imaging examination and biochemical examination, and the survival status was documented at each visit. Progression-free survival (PFS) and overall survival (OS) were evaluated based on follow-up records, with the last follow-up date of March 2020. Additionally, the median follow-up time in our study was $17(12-21)$ months. The PFS was defined as the time interval from the date of first DEBTACE to the date of disease progression; the OS was defined as the time interval from the date of first DEBTACE to the date of patient's death.

\section{Statistical Analysis}

SPSS 21.0 statistical software (IBM, Chicago, Illinois, USA) was used for data processing and statistical analysis. Quantitative data were described as mean \pm standard deviation (SD), and qualitative data were described as number with percentage (No. (\%)). Comparison of quantitative data between two groups was determined by Student's $t$ test, and comparison of qualitative data between two groups was determined by Chi-square test, Fisher's exact test or Wilcoxon rank sum test. PFS and OS were demonstrated using KaplanMeier curves, and the difference in PFS and OS between the two groups was determined by the Log rank test. Factors related to VL occurrence were assessed by multivariate logistic regression model analysis, and factors related to PFS and OS were analyzed by multivariate Cox's proportional hazards regression. 
$P$ value $<0.05$ was considered as statistically significant.

\section{Results}

\section{Characteristics of HCC Patients and the Treated Nodules}

In the $286 \mathrm{HCC}$ patients, the number of patients aged $<60$ years and patients aged $\geq 60$ years was $211(73.8 \%)$ and 75 (26.2\%), respectively (Table 1). There were 199 (69.6\%) males and $87(30.4 \%)$ females. In addition, the number of patients with unifocal tumors and patients with multifocal tumors was $205(71.7 \%)$ and $81(28.3 \%)$, respectively. The number of patients with portal vein invasion and patients with no portal vein invasion was $205(71.7 \%)$ and 81
$(28.3 \%)$, respectively. The number of patients in BCLC stage $\mathrm{A}, \mathrm{BCLC}$ stage $\mathrm{B}$ and $\mathrm{BCLC}$ stage $\mathrm{C}$ was 30 $(10.5 \%), 172(60.1 \%)$ and $84(29.4 \%)$, respectively. In addition, no difference was found between VL patients and nonVL patients regarding age, sex, etiology, tumor number, portal vein invasion, Child-Pugh stage, BCLC stage or loading drugs (all $P>0.05$ ). The other information of HCC patients' characteristics could be seen in Table 1 .

With regard to the treated nodules $(\mathrm{N}=384), 268(69.8 \%)$ nodules were located in the right liver, and $116(30.2 \%)$ nodules were located in the left liver (Table 2). The number of nodules with a size of $<3 \mathrm{~cm}, 3-5 \mathrm{~cm}, 5-10 \mathrm{~cm}$ and $\geq 10 \mathrm{~cm}$ was $26(6.8 \%), 58(15.1 \%), 295$ (76.8\%), and 5 (1.3\%), respectively. One hundred ninety-nine (51.8\%) nodules

Table I Clinical Characteristics of Patients

\begin{tabular}{|c|c|c|c|c|}
\hline Items & Total Patients $(\mathrm{N}=\mathbf{2 8 6})$ & VL Patients $(n=49)$ & Non-VL Patients $(n=237)$ & $P$ value \\
\hline Age, No. (\%) & & & & 0.681 \\
\hline$<60$ years & $211(73.8)$ & $35(71.4)$ & $176(74.3)$ & \\
\hline$\geq 60$ years & $75(26.2)$ & $14(28.6)$ & 61 (25.7) & \\
\hline Gender, No. (\%) & & & & 0.709 \\
\hline Male & $199(69.6)$ & $33(67.3)$ & $166(70.0)$ & \\
\hline Female & $87(30.4)$ & $16(32.7)$ & $71(30.0)$ & \\
\hline Etiology, No. (\%) & & & & 0.906 \\
\hline Hepatitis B & $183(64.0)$ & $33(67.3)$ & $150(63.3)$ & \\
\hline Hepatitis C & $5 \mathrm{I}(17.8)$ & $9(18.4)$ & $42(17.7)$ & \\
\hline Alcoholic cirrhosis & $31(10.8)$ & $4(8.2)$ & $27(11.4)$ & \\
\hline Alcohol/hepatitis & $4(1.4)$ & I (2.0) & $3(1.3)$ & \\
\hline Others & $17(6.0)$ & $2(4.1)$ & $15(6.3)$ & \\
\hline Tumor number, No. (\%) & & & & 0.966 \\
\hline Unifocal & $205(71.7)$ & $35(71.4)$ & $170(71.7)$ & \\
\hline Multifocal & $81(28.3)$ & $14(28.6)$ & $67(28.3)$ & \\
\hline Portal vein invasion, No. (\%) & & & & 0.151 \\
\hline No & 205 (7I.7) & $31(63.3)$ & $174(73.4)$ & \\
\hline Yes & $81(28.3)$ & $18(36.7)$ & $63(26.6)$ & \\
\hline Child-Pugh stage, No. (\%) & & & & 0.662 \\
\hline A & $173(60.5)$ & $31(63.3)$ & 142 (59.9) & \\
\hline B & $113(39.5)$ & $18(36.7)$ & $95(40.1)$ & \\
\hline BCLC stage, No. (\%) & & & & 0.359 \\
\hline A & $30(10.5)$ & $5(10.2)$ & $25(10.5)$ & \\
\hline B & $172(60.1)$ & $33(67.3)$ & $139(58.7)$ & \\
\hline C & $84(29.4)$ & II (22.5) & $73(30.8)$ & \\
\hline Loading drugs, No. (\%) & & & & 0.819 \\
\hline Doxorubicin & $156(54.5)$ & $26(53.1)$ & $130(54.9)$ & \\
\hline Arsenic trioxide & $130(45.5)$ & $23(46.9)$ & $107(45.1)$ & \\
\hline
\end{tabular}

Note: Comparison between the two groups (VL group and non-VL group) was determined by Chi-square test or Wilcoxon rank sum test. Abbreviations: VL, vascular lake; BCLC, Barcelona Clinic Liver Cancer. 
Table 2 Characteristics of Treated Nodules

\begin{tabular}{|c|c|c|c|c|}
\hline Items & Total Treated Nodules $(\mathbf{N}=\mathbf{3 8 4})$ & VL Nodules $(n=63)$ & Non-VL Nodules $(n=32 I)$ & $P$ value \\
\hline Tumor location, No. (\%) & & & & 0.771 \\
\hline Right & $268(69.8)$ & $43(68.3)$ & $225(70.1)$ & \\
\hline Left & $116(30.2)$ & $20(31.7)$ & $96(29.9)$ & \\
\hline Tumor size, No. (\%) & & & & 0.054 \\
\hline$<3 \mathrm{~cm}$ & $26(6.8)$ & $2(3.2)$ & $24(7.5)$ & \\
\hline $3-5 \mathrm{~cm}$ & $58(15.1)$ & $6(9.5)$ & $52(16.2)$ & \\
\hline $5-10 \mathrm{~cm}$ & $295(76.8)$ & $54(85.7)$ & $24 I(75.1)$ & \\
\hline$\geq 10 \mathrm{~cm}$ & $5(1.3)$ & I (I.6) & $4(1.2)$ & \\
\hline Pseudocapsule, No. (\%) & & & & $<0.001$ \\
\hline No & $199(51.8)$ & $15(23.8)$ & $184(57.3)$ & \\
\hline Yes & $185(48.2)$ & $48(76.2)$ & $137(42.7)$ & \\
\hline CalliSpheres ${ }^{\circledR}$ diameter & & & & 0.039 \\
\hline $100-300 \mu \mathrm{m}$ & $297(77.3)$ & $55(87.3)$ & $242(75.4)$ & \\
\hline $300-500 \mu \mathrm{m}$ & $87(22.7)$ & $8(12.7)$ & $79(24.6)$ & \\
\hline
\end{tabular}

Note: Comparison was determined by Chi-square test or Wilcoxon rank sum test. Abbreviation: VL, vascular lake.

presented with pseudocapsule and 185 (48.2\%) nodules did not present with pseudocapsule. Additionally, the number of nodules treated with 100-300 $\mu \mathrm{m}$ CalliSpheres ${ }^{\circledR}$ beads and 300-500 $\mu \mathrm{m}$ CalliSpheres ${ }^{\circledR}$ beads was 297 (77.3\%) and 87 (22.7\%), respectively. Moreover, there was no difference in tumor location $(P=0.771)$ or tumor size $(P=0.054)$ between VL nodules and non-VL nodules; however, the occurrence of pseudocapsule was elevated in VL nodules compared to nonVL nodules $(P<0.001)$, and the proportion of treatments with 100-300 $\mu \mathrm{m}$ CalliSpheres ${ }^{\circledR}$ beads was increased in VL nodules compared to non-VL nodules $(P=0.039)$.

\section{Incidence of VL and Related Factors}

The patient-based VL occurrence rate was $17.1 \%$ and the nodule-based VL occurrence rate was 16.4\% (Figure 2A). In addition, the postoperative hemoglobin level did not vary compared to the preoperative level, indicating that treatment of VL using PVA was tolerable $(P=0.342)$ (Figure 2B). Furthermore, multivariate logistic regression model analysis elucidated that larger tumor size $(P=$ 0.005), pseudocapsule $(P<0.001)$ and smaller bead size $(P=0.007)$ were independently associated with VL occurrence in HCC patients treated with DEB-TACE (Table 3).
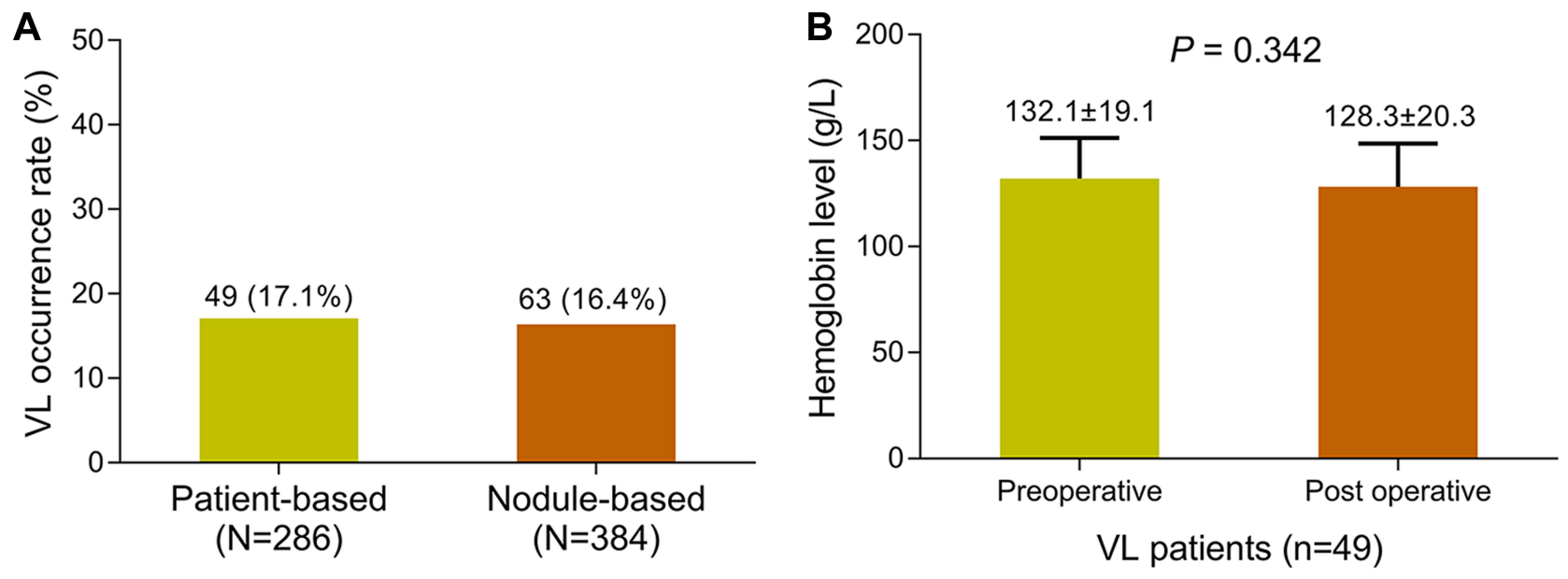

Figure 2 VL occurrence. VL occurrence rate in patients and nodules (A). Preoperative hemoglobin level and postoperative hemoglobin level (B). Abbreviation: VL, vascular lake. 
Table 3 Nodule-Based Analysis of Factors Related to VL Occurrence

\begin{tabular}{|c|c|c|c|c|}
\hline \multirow[t]{3}{*}{ Items } & \multicolumn{4}{|c|}{ Multivariate Logistic Regression Model } \\
\hline & \multirow[t]{2}{*}{$P$ value } & \multirow[t]{2}{*}{ OR } & \multicolumn{2}{|c|}{$95 \% \mathrm{Cl}$} \\
\hline & & & Lower & Higher \\
\hline Tumor size* & 0.005 & 2.417 & 1.303 & 4.482 \\
\hline Pseudocapsule (yes vs no) & $<0.001$ & 6.117 & 3.205 & 11.675 \\
\hline CalliSpheres ${ }^{\circledR}$ diameter $(100-300 \mu \mathrm{m}$ vs $300-500 \mu \mathrm{m})$ & 0.007 & 3.124 & 1.373 & 7.105 \\
\hline
\end{tabular}

Notes: *Tumor size was categorized as $<3 \mathrm{~cm}=1,3-5 \mathrm{~cm}=2,5-10 \mathrm{~cm}=3$ and $\geq 10 \mathrm{~cm}=4$. Factors affecting VL were analyzed by multivariate logistic regression model.

Abbreviations: VL, vascular lake; OR, odds ratio; $\mathrm{Cl}$, confidence interval.

In addition, the category of chemotherapy drugs used in our study was not correlated with VL occurrence in patients $(P=0.819)$ (Supplementary Table 1$)$.

\section{Correlation Between VL and Treatment Response}

In the total treated nodules, the rates of nodules assessed as CR, PR, SD and PD were 18.5\%, 42.4\%, 22.7\% and 16.4\%, respectively (Table 4). Moreover, the ORR and DCR were $60.9 \%$ and $83.6 \%$, respectively. In VL nodules, the CR, PR,
SD, PD, ORR and DCR were $22.2 \%, 66.7 \%, 9.5 \%, 1.6 \%$, $88.9 \%$ and $98.4 \%$, respectively, and the $\mathrm{CR}, \mathrm{PR}, \mathrm{SD}, \mathrm{PD}$, ORR and DCR in non-VL nodules were $17.8 \%, 37.7 \%$, $25.2 \%, 19.3 \%, 55.5 \%$ and $67.4 \%$, respectively. In addition, the total response rate $(P<0.001)$, ORR $(P<0.001)$ and DCR $(P<0.001)$ were all increased in VL nodules compared to non-VL nodules. In the total HCC patients, the percentages of patients who achieved CR, PR, SD and PD were $18.5 \%, 47.9 \%, 21.0 \%$ and $12.6 \%$, and the ORR and DCR were $66.4 \%$ and 87.4 , respectively (Table 5). In VL

Table 4 Nodule-Based Analysis of Treatment Response After First DEB-TACE Therapy

\begin{tabular}{|c|c|c|c|c|}
\hline Items & Total Treated Nodules $(\mathbf{N}=384)$ & VL Nodules $(n=63)$ & $\begin{array}{l}\text { Non-VL Nodules } \\
\qquad(n=321)\end{array}$ & $P$ value \\
\hline Total response & & & & $<0.001$ \\
\hline CR, No. (\%) & $7 \mid(18.5)$ & $14(22.2)$ & $57(\mid 7.8)$ & \\
\hline PR, No. (\%) & $163(42.4)$ & $42(66.7)$ & 121 (37.7) & \\
\hline SD, No. (\%) & $87(22.7)$ & $6(9.5)$ & $81(25.2)$ & \\
\hline PD, No. (\%) & $63(16.4)$ & I (I.6) & $62(19.3)$ & \\
\hline ORR, No. (\%) & $234(60.9)$ & $56(88.9)$ & $178(55.5)$ & $<0.001$ \\
\hline DCR, No. (\%) & $321(83.6)$ & $62(98.4)$ & $259(67.4)$ & $<0.001$ \\
\hline
\end{tabular}

Note: Comparison was determined by Wilcoxon rank test or Chi-square test.

Abbreviations: DEB-TACE, drug-eluting beads transarterial chemoembolization; VL, vascular lake; CR, complete response; PR, partial response; SD, stable disease; PD, progressive disease; ORR, objective response rate; DCR, disease control rate.

Table 5 Patient-Based Analysis of Treatment Response After First DEB-TACE Therapy

\begin{tabular}{|c|c|c|c|c|}
\hline Items & Total Patients ( $\mathbf{N = 2 8 6 )}$ & VL Patients $(n=49)$ & Non-VL Patients $(n=237)$ & $P$ value \\
\hline Total response & & & & 0.002 \\
\hline CR, No. (\%) & $53(18.5)$ & $10(20.4)$ & $43(18.2)$ & \\
\hline PR, No. (\%) & 137 (47.9) & $35(7 I .4)$ & $102(43.0)$ & \\
\hline SD, No. (\%) & $60(21.0)$ & $3(6.2)$ & $57(24.0)$ & \\
\hline PD, No. (\%) & $36(12.6)$ & I (2.0) & $35(14.8)$ & \\
\hline ORR, No. (\%) & $190(66.4)$ & $45(91.8)$ & $145(6 \mid .2)$ & $<0.001$ \\
\hline DCR, No. (\%) & $250(87.4)$ & $48(98.0)$ & $202(85.2)$ & 0.014 \\
\hline
\end{tabular}

Note: Comparison was determined by Wilcoxon rank test or Chi-square test.

Abbreviations: DEB-TACE, drug-eluting beads transarterial chemoembolization; VL, vascular lake; CR, complete response; PR, partial response; SD, stable disease; PD, progressive disease; ORR, objective response rate; DCR, disease control rate. 
HCC patients, the CR, PR, SD, PD, ORR and DCR were $20.4 \%, 71.4 \%, 6.2 \%, 2.0 \%, 91.8 \%$ and $98.0 \%$, respectively. In non-VL HCC patients, the CR, PR, SD, PD, ORR and DCR were $18.2 \%, 43.0 \%, 24.0 \%, 14.8 \%, 61.2 \%$ and $85.2 \%$, respectively. The total response rate $(P=0.002)$, ORR $(P<$ $0.001)$ and DCR $(P=0.014)$ were all elevated in VL HCC patients compared to non-VL HCC patients.

\section{Correlation Between VL and Survival}

PFS $(P<0.001)$ (Figure 3A) was more favorable in VL HCC patients than in non-VL HCC patients, and OS $(P<$ 0.001) (Figure 3B) was also more prolonged in VL HCC patients than in non-VL HCC patients. Furthermore, multivariate Cox's proportional hazards regression analysis showed that VL was an independent factor for predicting longer PFS $(P<0.001)$ and OS $(P<0.001)$ (Table 6$)$. However, portal vein invasion was independently correlated with worse PFS $(P<0.001)$ and OS $(P<0.001)$.

\section{Correlation Between VL and Liver Function}

Between VL patients and non-VL patients, the ALT levels before $(P=0.711), 1$ day after $(P=0.364), 7$ days after $(P=0.534)$, and 1 month after $(P=0.152)$ DEB-TACE was of no difference (Table 7). The AST levels before $(P=$ $0.082), 1$ day after $(P=0.832), 7$ days after $(P=0.223)$ and 1 month after $(P=0.581)$ DEB-TACE did not vary between VL patients and non-VL patients. In addition, the
ALB levels before $(P=0.577), 1$ day after $(P=0.212), 7$ days after $(P=0.433)$ and 1 month after $(P=0.579)$ DEBTACE were similar between VL patients and non-VL patients. These data suggested that VL did not result in decreased liver function in HCC patients receiving DEBTACE.

\section{Correlation Between VL and Adverse Events}

The incidences of adverse events, including fever $(P=$ $0.171)$, pain $(P=0.199)$, ascites $(P=0.681)$, nausea and vomiting $(P=0.130)$, and gastrointestinal bleeding $(P=$ 1.000) were of no difference between VL patients and non-VL patients (Table 8). Moreover, the majority of the adverse events were grade 1 and grade 2, and a small proportion of adverse events were grade 3 . In addition, no grade 4 adverse events existed.

\section{Discussion}

More than a decade has passed since the first application of DEB-TACE in the treatment of HCC patients (mostly middle-stage patients), and newer data suggest that it might also be applicable for other categories of patients, including its use in early-stage patients before surgery as bridge therapy, advanced patients and patients with liver metastasis. $^{17-19}$ Thus, further investigation of DEB-TACE in a wider group of HCC patients is warranted. For HCC, mounting evidence has shown that DEB-TACE is efficient

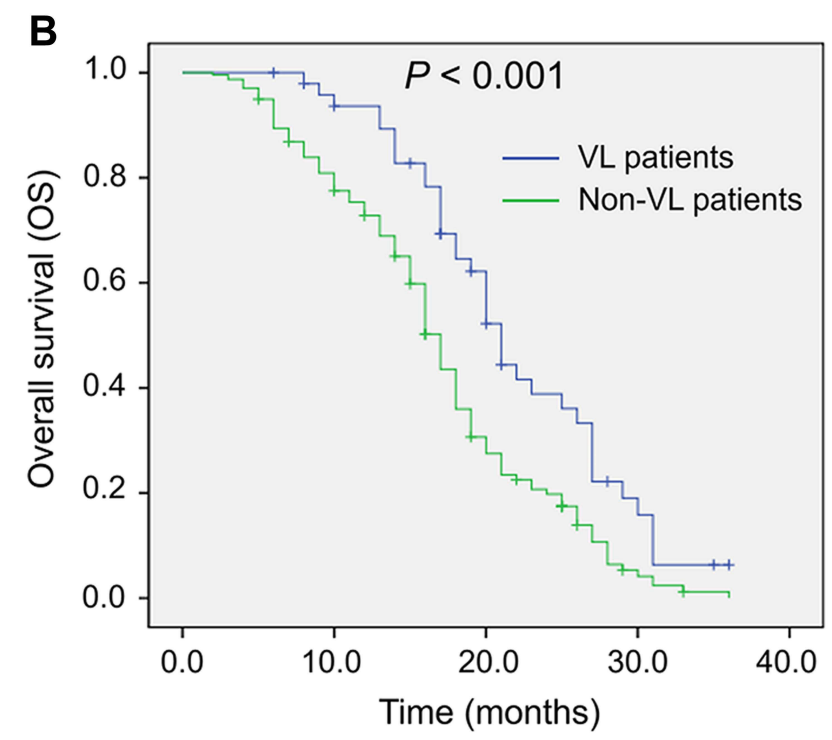

Figure 3 Comparison of PFS and OS between VL patients and non-VL patients. Comparison of PFS between VL patients and non-VL patients (A). Comparison of OS between $V L$ patients and non-VL patients (B).

Abbreviations: PFS, progression-free survival; OS, overall survival; VL, vascular lake. 
Table 6 Multivariate Cox's Proportional Hazards Regression Analysis of Factors Related to PFS and OS

\begin{tabular}{|l|l|l|l|l|}
\hline \multirow{2}{*}{ Items } & \multicolumn{2}{|c|}{ PFS } & \multicolumn{2}{c|}{ OS } \\
\cline { 2 - 5 } & P value & HR (95\% Cl) & P value & HR (95\% Cl) \\
\hline Non-VL vs VL & $<0.001$ & $1.988(1.389-2.847)$ & $<0.001$ & $4.442(2.938-6.717)$ \\
Portal vein invasion (yes vs no) & $<0.001$ & $2.714(2.034-3.621)$ & $<0.001$ & $4.815(3.44 I-6.739)$ \\
\hline
\end{tabular}

Abbreviations: PFS, progression-free survival; OS, overall survival; VL, vascular lake; HR, hazard ratio; Cl, confidence interval.

Table 7 Liver Function Indexes Before and After First DEB-TACE Therapy

\begin{tabular}{|c|c|c|c|}
\hline Liver Function Indexes & VL Patients $(n=49)$ & Non-VL Patients $(n=237)$ & $P$ value \\
\hline \multicolumn{4}{|l|}{$\operatorname{ALT}(\mathrm{U} / \mathrm{L})$, mean $\pm \mathrm{SD}$} \\
\hline Before DEB-TACE & $47.4 \pm 41.2$ & $49.7 \pm 29.1$ & 0.711 \\
\hline I day after DEB-TACE & $|7| .5 \pm 87.2$ & $182.9 \pm 78.4$ & 0.364 \\
\hline 7 days after DEB-TACE & $75.2 \pm 33.6$ & $71.4 \pm 39.9$ & 0.534 \\
\hline I month after DEB-TACE & $39.1 \pm 18.9$ & $45.9 \pm 32.0$ & 0.152 \\
\hline \multicolumn{4}{|l|}{ AST (U/L), mean $\pm S D$} \\
\hline Before DEB-TACE & $49.50 \pm 27.2$ & $44.2 \pm 21.7$ & 0.082 \\
\hline I day after DEB-TACE & $163.6 \pm 56.5$ & $161.7 \pm 67.2$ & 0.832 \\
\hline 7 days after DEB-TACE & $81.1 \pm 36.1$ & $89.4 \pm 44.7$ & 0.223 \\
\hline I month after DEB-TACE & $57.4 \pm 35.1$ & $54.1 \pm 38.7$ & $0.58 \mathrm{I}$ \\
\hline \multicolumn{4}{|l|}{$\operatorname{ALB}(g / L)$, mean $\pm S D$} \\
\hline Before DEB-TACE & $35.7 \pm 4.9$ & $36.1 \pm 4.5$ & 0.577 \\
\hline I day after DEB-TACE & $31.9 \pm 5.5$ & $30.9 \pm 5.0$ & 0.212 \\
\hline 7 days after DEB-TACE & $33.4 \pm 5.2$ & $32.8 \pm 4.8$ & 0.433 \\
\hline I month after DEB-TACE & $37.5 \pm 5.0$ & $37.9 \pm 4.5$ & 0.579 \\
\hline
\end{tabular}

Note: Comparison was determined by Student's $t$ test.

Abbreviations: DEB-TACE, drug-eluting beads transarterial chemoembolization; VL, vascular lake; ALT, alanine aspartate aminotransferase; SD, standard deviation; AST, aspartate aminotransferase; ALB, albumin.

and tolerable and that it is superior to cTACE in HCC patients. For instance, a recent multicenter cohort study has revealed that DEB-TACE realizes better CR and ORR as well as similar liver function laboratory index levels and adverse event incidences compared to cTACE in HCC patients. ${ }^{20}$ Another retrospective cohort study has elucidated that the incidences of adverse events, including abdominal pain, nausea and vomiting, fever and fatigue, are markedly reduced in HCC patients treated with DEBTACE compared to patients treated with cTACE. ${ }^{21}$

Apart from the favorable efficiency and safety of DEBTACE in HCC patients, other aspects have rarely been investigated, such as the role of VL during the DEB-TACE procedure. Therefore, the present study was conducted and it displayed that the incidence of VL was $17.1 \%$ in HCC patients and $16.4 \%$ in treated nodules during the DEBTACE procedure. A previous retrospective cohort study conducted in HCC patients treated with cTACE reports cases of VL that occurred before embolization with an incidence of
$21.9 \%$ in patients (151 HCC patients) and $16.8 \%$ in all treated nodules (232 treated nodules). ${ }^{12}$ With regard to VL during DEB-TACE, a previous cohort study illuminates that in HCC patients who have no vascular invasion or metastasis treated with DEB-TACE, the incidence of VL is $26.0 \%$ in the total treated nodules and that the VL is subsequently managed with gelatin sponge particles until it disappears. ${ }^{12}$ In addition, a retrospective cohort study illustrates that VL occurs in $12.1 \%$ all treated nodules (there are 323 treated nodules in $200 \mathrm{HCC}$ patients) during DEB-TACE treatment. ${ }^{13}$ Compared with these previous studies, the VL incidence in our study was slightly different in number but was within the range of the previous reports regarding VL incidence during TACE treatment. The slight difference may be attributed to the variance in sample size, patient clinicopathological features and treatment modalities. With regard to the mechanisms responsible for the occurrence of VL during DEB-TACE treatment in our HCC patients, there is still a lack of established evidence. One possible explanation 
Table 8 Adverse Events After First DEB-TACE Therapy

\begin{tabular}{|c|c|c|c|}
\hline Items & $\begin{array}{c}\text { VL } \\
\text { Patients } \\
(n=49)\end{array}$ & $\begin{array}{l}\text { Non-VL } \\
\text { Patients } \\
(n=237)\end{array}$ & $P$ value \\
\hline Fever, No. (\%) & $21(42.9)$ & $127(53.6)$ & 0.171 \\
\hline Grade I & $16(32.7)$ & $92(38.8)$ & \\
\hline Grade 2 & $5(10.2)$ & 33 (13.9) & \\
\hline Grade 3 & $0(0.0)$ & $2(0.8)$ & \\
\hline Pain, No. (\%) & $26(53.1)$ & $102(43.0)$ & 0.199 \\
\hline Grade I & $7(14.3)$ & $30(12.7)$ & \\
\hline Grade 2 & $15(30.6)$ & $58(24.5)$ & \\
\hline Grade 3 & $4(8.2)$ & $14(5.9)$ & \\
\hline $\begin{array}{l}\text { Ascites ( } 4 \text { weeks after } \\
\text { DEB-TACE), No. (\%) }\end{array}$ & $14(28.6)$ & 61 (25.7) & $0.68 I$ \\
\hline Grade I & $10(20.4)$ & 47 (19.8) & \\
\hline Grade 2 & $3(6.1)$ & $9(3.8)$ & \\
\hline Grade 3 & I (2.0) & $5(2.1)$ & \\
\hline $\begin{array}{l}\text { Nausea and vomiting, } \\
\text { No. (\%) }\end{array}$ & $8(16.3)$ & $63(26.6)$ & 0.130 \\
\hline Garde I & $8(16.3)$ & $63(26.6)$ & \\
\hline Grade 2 & $0(0.0)$ & $0(0.0)$ & \\
\hline Grade 3 & $0(0.0)$ & $0(0.0)$ & \\
\hline $\begin{array}{l}\text { Gastrointestinal } \\
\text { bleeding, No. (\%) }\end{array}$ & $0(0.0)$ & I $(0.4)$ & 1.000 \\
\hline Grade I & $0(0.0)$ & I $(0.4)$ & \\
\hline Grade 2 & $0(0.0)$ & $0(0.0)$ & \\
\hline Grade 3 & $0(0.0)$ & $0(0.0)$ & \\
\hline
\end{tabular}

Note: Comparison was determined by Fisher's exact test or Chi-square test. Abbreviations: VL, vascular lake; DEB-TACE, drug-eluting beads transarterial chemoembolization.

is that the microvasculature is often weak in HCC tumors, hence, when injecting the contrast agent, normal saline and DEBs are into the tumor-feeding artery, the microvasculature is easily broken due to the sudden increase in pressure, which will consequently cause the VL during DEB-TACE. ${ }^{12}$

Furthermore, several factors related to $\mathrm{VL}$ occurrence during DEB-TACE were identified in our study, including larger tumor size, pseudocapsule and smaller bead size. A retrospective cohort study elucidates that poor tumor differentiation is an independent predictive factor for the occurrence of VL in $\mathrm{HCC}$ patients who receive cTACE. ${ }^{22}$ Another cohort study reveals that in HCC patients treated with DEBTACE, tumor size larger than $3 \mathrm{~cm}$, the presence of a pseudocapsule, and higher alpha fetal protein (AFP) levels are independently associated with the occurrence of VL. ${ }^{13}$ These two prior studies present VL-related factors similar to ours. With regard to the factors independently correlated with VL during DEB-TACE in our study, their correlation with
VL may be explained by the following theories: (1) larger tumor size: we presumed that the VL was more likely to occur in a larger tumor because more microspheres would be used if the tumor was larger, which increased the risk of VL due to an increase pressure in the vascular bed. (2) Pseudocapsule: the existence of them in a tumor mostly resulted from the tumor growth causing pressure on the surrounding tissue, which mainly composed of fibrous tissue and liver tissue (if the pseudocapsule occurred in a liver cancer), and this could prevent the growth of tumor to some extent. Therefore, we presumed that pseudocapsule might enhance the embolization effect during DEB-TACE and contribute to the occurrence of VL. However, this is merely a presumption that should be validated by animal experiments in the future. (3) With regard to smaller bead size, smaller microbeads more easily entered the microvasculature; therefore, the accumulation of smaller microbeads elevated the pressure inside the vessels, led to the rupture of vessels and/or targeted tumors, resulting in VL occurrence.

In this study, we also found that VL occurrence was associated with a more favorable treatment response and was an independent predictive factor for prolonged DFS and OS in HCC patients. Predictive factors other than VL have been evaluated by previous studies, such as, a cohort study elucidates that vascular endothelial growth factor (VEGF) level could be a prognostic factor in unresectable HCC patients receiving DEB-TACE and cTACE. $^{23}$ As for VL, a retrospective cohort study reported that the occurrence of VL is correlated with better ORR in HCC patients who are treated with DEB-TACE, while, the association of VL with survival is not investigated in this study. ${ }^{13}$ Another retrospective cohort study shows that in advanced HCC patients (BCLC C stage) treated with DEB-TACE, the occurrence of VL is independently associated with more favorable radiological response, and the other independent predictive factor is homogeneous tumor enhancement on cone-beam computed tomography; however, this previous study predominantly aims at exploring the predictive factors for treatment responses but not the correlation between VL and long-term patient prognosis. ${ }^{24}$ These prior investigations on the prognostic role of VL in $\mathrm{HCC}$ patients treated with DEB-TACE are quite limited in the literature, and the elemental mechanism has not yet been studied. More importantly, these previous studies are in accordance with ours regarding the correlation of VL with better outcomes in HCC patients treated with DEB-TACE. With regard to our finding, it not only uncovered the correlation of VL during DEB-TACE with a better treatment response but also its association with a more favorable long-term survival. As for 
possible explanations, these results might derive from the fact that the occurrence of VL indicates a better embolization of the tumor-feeding artery; in addition, tumor rupture, a cause of $\mathrm{VL}$, also indicates a better treatment effect of DEB-TACE. ${ }^{12}$ Nevertheless, the detailed underlying mechanism should be explored by further experiments. However, the results still provide valuable information that VL may be a prognostic factor in the management of HCC patients treated with DEBTACE. Moreover, we also discovered that the occurrence of VL was not correlated with more liver function damage or adverse events in HCC patients treated with DEB-TACE. This result probably indicates that VL has no impact on the safety of DEB-TACE in HCC patients, which, however, needs to be validated by more large-scale studies and experimental studies.

Compared with previous studies, one predominant advantage of the present study was that the sample size was larger, which was beneficial for a more sufficient statistical power. However, there were also some limitations in this study. First, the percentage of patients or nodules with VL was small, which might interfere with the statistical analysis; however, this was normal because VL was rare in the clinical setting. Second, we included only HCC patients and not patients with other types of liver cancer, suggesting that our findings may not be applicable to patients with other liver cancers. Third, the patients with portal vein invasion were included in our study, however, TACE is recommended as standard therapy for BCLC stage B patients but not BCLC stage $\mathrm{C}$ patients (with portal vein invasion). Nonetheless, in the clinical setting, TACE is applied in patients with portal vein invasion due to that these patients do not have too many treatment options, and there is also study reporting that TACE could be applied in patients with portal vein invasion. ${ }^{10}$ Hence, we included the patients with portal vein invasion in order to include as many patients as possible.

In conclusion, VL occurs in $17.1 \%$ HCC patients treated with DEB-TACE, and it is correlated with larger tumor size, pseudocapsule, smaller bead size, more favorable treatment response as well as better survival.

\section{Data Sharing Statement}

The data would be accessible via contacting the corresponding author (for scientific research use only).

\section{Acknowledgments}

This study was supported by China Health Promotion Foundation (No. XM_2018_011_0006_01) and Henan
Medical Science and Technology Research Project (LHGJ2019162). Hao Li and Manzhou Wang are co-first authors.

\section{Disclosure}

The authors declare that they have no conflicts of interest.

\section{References}

1. World Health Organization. Projections of mortality and causes of death, 2016 to 2060. Available from: http:/www.who.int/healthinfo/ global_burden_disease/projections/en/. Accessed May 03, 2021.

2. $\mathrm{Xu}$ J. Trends in liver cancer mortality among adults aged 25 and over in the United States, 2000-2016. Hyattsville (MD): National Center for Health Statistics; 2018.

3. Tsilimigras DI, Bagante F, Moris D, et al. Defining the chance of cure after resection for hepatocellular carcinoma within and beyond the Barcelona Clinic Liver Cancer guidelines: a multi-institutional analysis of 1010 patients. Surgery. 2019;166(6):967-974. doi:10.1016/j.surg.2019.08.010

4. Pinero F, Marciano S, Fernandez N, et al. Adherence to Barcelona Clinic Liver Cancer therapeutic algorithm for hepatocellular carcinoma in the daily practice: a multicenter cohort study from Argentina. Eur J Gastroenterol Hepatol. 2018;30(4):376-383. doi:10.1097/MEG.0000000000001049

5. Oweira H, Petrausch U, Helbling D, et al. Early stage hepatocellular carcinoma in the elderly: a SEER database analysis. J Geriatr Oncol. 2017;8(4):277-283. doi:10.1016/j.jgo.2017.03.002

6. Akada K, Koyama N, Taniguchi S, et al. Database analysis of patients with hepatocellular carcinoma and treatment flow in early and advanced stages. Pharmacol Res Perspect. 2019;7(4):e00486. doi:10.1002/prp2.486

7. Hartke J, Johnson M, Ghabril M. The diagnosis and treatment of hepatocellular carcinoma. Semin Diagn Pathol. 2017;34(2):153-159. doi:10.1053/j.semdp.2016.12.011

8. Li H, Wu F, Duan M, et al. Drug-eluting bead transarterial chemoembolization (TACE) vs conventional TACE in treating hepatocellular carcinoma patients with multiple conventional TACE treatments history: a comparison of efficacy and safety. Medicine (Baltimore). 2019;98(21):e15314. doi:10.1097/MD.0000000000015314

9. Xiang H, Long L, Yao Y, et al. CalliSpheres drug-eluting bead transcatheter arterial chemoembolization presents with better efficacy and equal safety compared to conventional TACE in treating patients with hepatocellular carcinoma. Technol Cancer Res Treat. 2019;18:1533033819830751. doi:10.1177/1533033819830751

10. Prajapati HJ, Dhanasekaran R, El-Rayes BF, et al. Safety and efficacy of doxorubicin drug-eluting bead transarterial chemoembolization in patients with advanced hepatocellular carcinoma. J Vasc Interv Radiol. 2013;24(3):307-315. doi:10.1016/j.jvir.2012.11.026

11. Liu J, Xu J, Zhang W, et al. Safety and efficacy of drug-eluting bead transarterial chemoembolization combined with apatinib in patients with advanced hepatocellular carcinoma. Acad Radiol. 2020;27 (5):704-709. doi:10.1016/j.acra.2019.07.003

12. Seki A, Hori S, Shimono C. Management of vascular lake phenomenon on angiography during chemoembolization with superabsorbent polymer microspheres. Jpn J Radiol. 2015;33(12):741-748. doi:10.1007/s11604-015-0486-2

13. Cavalcante RN, Nasser F, Motta-Leal-Filho JM, et al. Occurrence of vascular lake phenomenon as a predictor of improved tumor response in hec patients that underwent DEB-TACE. Cardiovasc Intervent Radiol. 2017;40(7):1044-1051. doi:10.1007/s00270-017-1678-1 
14. Lencioni R, Llovet JM. Modified RECIST (mRECIST) assessment for hepatocellular carcinoma. Semin Liver Dis. 2010;30(1):52-60. doi:10.1055/s-0030-1247132

15. Liang B, Zhao D, Liu Y, et al. Chemoembolization of liver cancer with doxorubicin-loaded CalliSpheres microspheres: plasma pharmacokinetics, intratumoral drug concentration, and tumor necrosis in a rabbit model. Drug Deliv Transl Res. 2020;10(1):185-191. doi:10.1007/ s13346-019-00672-9

16. Duan XH, Li H, Ren JZ, et al. Hepatic arterial chemoembolization with arsenic trioxide eluting callispheres microspheres versus lipiodol emulsion: pharmacokinetics and intratumoral concentration In A rabbit liver tumor model. Cancer Manag Res. 2019;11:9979-9988. doi:10.2147/CMAR.S199188

17. Zhou Y. Drug-eluting bead transarterial chemoembolization is efficient and well-tolerated in treating elderly Chinese hepatocellular carcinoma patients. Int J Clin Exp Pathol. 2018;11(10):4867-4878.

18. Cao WZ, Zhou ZQ, Jiang S, et al. Efficacy and safety of drug-eluting beads for transarterial chemoembolization in patients with advanced hepatocellular carcinoma. Exp Ther Med. 2019;18(6):4625-4630. doi:10.3892/etm.2019.8163

19. Rostas J, Tam A, Sato T, et al. Image-guided transarterial chemoembolization with drug-eluting beads loaded with doxorubicin (DEBDOX) for unresectable hepatic metastases from melanoma: technique and outcomes. Cardiovasc Intervent Radiol. 2017;40 (9):1392-1400. doi:10.1007/s00270-017-1651-z
20. Ma Y, Zhao C, Zhao H, et al. Comparison of treatment efficacy and safety between drug-eluting bead transarterial chemoembolization with CalliSpheres((R)) microspheres and conventional transarterial chemoembolization as first-line treatment in hepatocellular carcinoma patients. Am J Transl Res. 2019;11(12):7456-7470.

21. Karalli A, Teiler J, Haji M, et al. Comparison of lipiodol infusion and drug-eluting beads transarterial chemoembolization of hepatocellular carcinoma in a real-life setting. Scand J Gastroenterol. 2019;54 (7):905-912. doi:10.1080/00365521.2019.1632925

22. Hu B, Zhong BY, Zhang L, et al. Occurrence of vascular lake phenomenon before embolization for the prediction of lipiodol uptake for intermediate-stage hepatocellular carcinoma patients that underwent cTACE. Cardiovasc Intervent Radiol. 2020;43:1460-1467. doi:10.1007/s00270-020-02501-w

23. Farid K, Elalfy H, Abo El-Khair SM, et al. Prognostic value of vascular endothelial growth factor in both conventional and drug eluting beads transarterial chemoembolization for treatment of unresectable hepatocellular carcinoma in $\mathrm{HCV}$ patients. Expert Rev Gastroenterol Hepatol. 2020;14(12):1203-1214. doi:10.1080/ 17474124.2020.1823215

24. Chang KH, Hwang ZA, Chang PY, et al. Predictive imaging for tumor response to drug-eluting microsphere transarterial chemoembolization in patients with BCLC-C advanced hepatocellular carcinoma. Sci Rep. 2019;9(1):20032. doi:10.1038/s41598-019$56545-1$
OncoTargets and Therapy

\section{Publish your work in this journal}

OncoTargets and Therapy is an international, peer-reviewed, open access journal focusing on the pathological basis of all cancers, potential targets for therapy and treatment protocols employed to improve the management of cancer patients. The journal also focuses on the impact of management programs and new therapeutic

\section{Dovepress}

agents and protocols on patient perspectives such as quality of life, adherence and satisfaction. The manuscript management system is completely online and includes a very quick and fair peer-review system, which is all easy to use. Visit http://www.dovepress.com/ testimonials.php to read real quotes from published authors. 